presumably through its involvement in Hedgehog signalling. Ongoing analysis will further define the mechanisms through which HHIPL1 contributes to atherosclerosis.

\section{B DKK3 STABILISES ATHEROSCLEROTIC PLAQUE VIA PROMOTING VASCULAR PROGENITOR AND FIBROBLAST DIFFERENTIATION TO SMOOTH MUSCLE CELLS}

${ }^{1}$ Eirini Karamariti, ${ }^{2}$ Chungang Zhai, ${ }^{1}$ Baoqi Yu, ${ }^{3}$ Christof Niehrs, ${ }^{1}$ Yanhua $\mathrm{Hu}$ ${ }^{2}$ Cheng Zhang, 'Q Qingbo Xu. 'Cardiovascular Division, King's College London BHF Centre, London, UK; ${ }^{2}$ The Key Laboratory of Cardiovascular Remodelling and Function Research, Chinese Ministry of Education and Chinese Ministry of Health, Qilu Hospital, Shandong University, Jinan, China; ${ }^{3}$ Institute of Molecular Biology (IMB), Mainz, Germany

\subsection{6/heartjnl-2016-309890.221}

Atherosclerosis, a chronic condition that can be converted into an acute clinical event caused by plaque rupture and thrombosis, has been the primary cause of mortality and morbidity worldwide. Dickkopf 3 (DKK3), a 36-kD secreted glycoprotein, has a role in cell differentiation, but little is known about its involvement in vascular disease. In the present study, we utilised a murine model of atherosclerosis $\left(\mathrm{ApoE}^{-1-}\right)$ in conjunction with $\mathrm{DKK}^{-/-}$to assess the effects of DKK3 on plaque stability.

We found that the absence of DKK3 leads to vulnerable unstable atherosclerotic plaques, due to a reduced number of smooth muscle cells (SMCs) and reduced matrix protein deposition, as well as increased haemorrhage and macrophage infiltration. Using a cell linear tracing method, vascular progenitors and fibroblasts from SM22-LacZ transgenic mice were isolated and applied to the adventitial side of injured femoral arteries resulting in migration of both types of cells to the intima. Upon migration the cells displayed beta-gal positivity, indicating SMC differentiation.

Further in vitro studies revealed that DKK3 can induce differentiation of Sca1+ vascular progenitors and fibroblasts into SMCs via activation of the TGF $\beta / A T F 6$ and Wnt signalling pathways. Finally, we assessed the therapeutic potential of DKK3 in mouse and rabbit models and found that DKK3 increases atherosclerotic plaque stability via an increase in SMC numbers and reduced vascular inflammation. Cumulatively, we provide the first evidence that DKK3 is a potent SMC differentiation factor, which may have a therapeutic effect in reducing acute haemorrhagic conditions through promotion of atherosclerotic plaque stability.

\section{NOX4-DEPENDENT REPROGRAMMING OF GLUCOSE METABOLISM AND FATTY ACID OXIDATION FACILITATES CARDIAC ADAPTION TO CHRONIC PRESSURE-OVERLOAD}

\begin{abstract}
${ }^{1}$ Adam Nabeebaccus*, ${ }^{2}$ Anne Hafstad, ${ }^{1}$ Anna Zoccarato, ${ }^{1}$ Tom Eykyn, ${ }^{3}$ James West, ${ }^{3} J u l e s$ Griffin, ${ }^{1}$ Manuel Mayr, ${ }^{1}$ Ajay Shah. ${ }^{1}$ King's College London, Cardiovascular Division and King's BHF Centre of Research Excellence; ' University of Tromsø, Cardiovascular Research Group; ${ }^{3}$ University of Cambridge, Department of Biochemistry, MRC Human Nutrition Research
\end{abstract}

\subsection{6/heartjnl-2016-309890.222}

Introduction Increased reactive oxygen species (ROS) production is involved in the pathophysiology of cardiac hypertrophy and failure. Interestingly, a specialised ROS-generating enzyme
NADPH oxidase-4 (Nox4) was previously found to have beneficial effects by promoting adaptive remodelling during pressure-overload cardiac hypertrophy. Nox4 modulates intracellular signalling cascades but how it achieves beneficial effects in the chronically overloaded heart remains unclear.

Methods and results To obtain an unbiased global overview of putative Nox4-mediated changes, the proteome of cardiac-specific Nox4 transgenic (TG) and wild-type (WT) mouse hearts was first characterised through a 2D-DIGE approach. TG hearts had a significant over-representation of changes in protein levels of enzymes involved in glucose and fatty acid utilisation. We therefore analysed the metabolome using $1 \mathrm{H}$ NMR and targeted LC-MS approaches. This identified a differential accumulation of glycolytic intermediates in the proximal part of glycolysis both in unstressed and pressureoverloaded TG hearts, as well as an increase in alanine levels (1.4 fold, $\mathrm{p}=0.05$ ), confirming significant alterations to metabolism. To specifically quantify glucose uptake, glycolysis, glucose oxidation and fatty acid oxidation rates, ex vivo working heart studies were conducted. TG hearts had a marked increase cf. WT in palmitate oxidation rate in the unstressed as well as pressure-overloaded heart (3.6 fold increase; $n=6$ / group; $\mathrm{p}=0.01)$. Glucose uptake was unaltered but glycolysis and oxidation rates were decreased, suggesting diversion of glucose away from oxidation. Importantly, an increase in palmitate oxidation was not detrimental either for in vivo cardiac energetics (31P-NMR) or contractile function during pressureoverload hypertrophy. We found that activity of the hexosamine biosynthesis pathway (HBP), an alternative route for glucose metabolism, was increased in TG hearts as assessed by the O-GlcNAc post-translational modification of cardiac proteins by $\mathrm{N}$-acetylglucosamine, the end-product of HBP. OGlcNAc levels were 2.4 fold higher in TG cf. WT $(n=4 /$ group; $\mathrm{p}=0.02$ ). In cultured cardiomyocytes, endogenous Nox4 induced similar changes in HBP and palmitate oxidation (extracellular flux analysis), and it was found that changes in O-GlcNAcylation regulated fatty acid oxidation.

Discussion These results show that Nox4 reprograms substrate utilisation in the heart by directing glucose towards the HBP and inducing a linked increase in fatty acid oxidation. These changes appear to enable the heart to better adapt to chronic pressure overload and may be important in the beneficial effects of Nox4 on cardiac remodelling. These data identify a novel redox mechanism that drives beneficial metabolic reprogramming in the heart and suggest potential new therapeutic approaches to promote adaptation to chronic overload stress.

\section{IMPACT OF HIGH-FLOW OXYGEN ON PERFUSION, MICROVASCULAR AND CAPILLIARY FUNCTION IN NORMAL VOLUNTEERS AND PATIENTS WITH CORONARY ARTERY DISEASE: A CARDIOVASCULAR MAGNETIC RESONANCE AND INVASIVE CORONARY PHYSIOLOGY STUDY}

${ }^{1}$ David P Ripley, ${ }^{1}$ Ripley DP, ${ }^{1}$ Broadbent DA, ${ }^{2}$ Corcoran D, ${ }^{1}$ Foley JRJ, ${ }^{3}$ Higgins DM, ${ }^{1}$ Plein S, ${ }^{2}$ Berry C, ${ }^{1}$ John P Greenwood. 'Multidisciplinary Cardiovascular Research Centre (MCRC) \& Leeds Institute of Genetics, Health and Therapeutics, University of Leeds, Leeds, UK; ${ }^{2} B H F$ Glasgow Cardiovascular Research Centre, Institute of Cardiovascular and Medical Sciences, University of Glasgow, Glasgow, UK; ${ }^{3}$ Philips Healthcare, Philips Centre, Guildford, Surrey, UK

10.1136/heartjnl-2016-309890.223 
Introduction Supplemental oxygen has long been used as a therapeutic agent in the management of ischaemic chest pain, but without a sound scientific basis. We proposed to investigate the effect of high-flow oxygen on coronary physiology, invasively and non-invasively, in those with severe coronary artery disease (CAD).

Methods 30 volunteers and 30 patients with severe CAD were allocated to receive high-flow air and oxygen in a random blinded order. They underwent cardiovascular magnetic resonance (CMR) T2* blood-oxygen level dependent (BOLD) and dynamic contrast-enhanced perfusion imaging. Myocardial blood flow (MBF), perfusion reserve (MPR), first-pass extraction fraction $(\mathrm{E})$, blood volume fraction $\left(\mathrm{v}_{\mathrm{b}}\right)$ and capillary permeability-surface area product (PS) were quantified using bookend $\mathrm{T}_{1}$ based non-linearity correction and distributed parameter model constrained deconvolution.

Invasive coronary physiology measurements were performed in severe CAD patients (Fractional Flow Reserve <0.75), also randomised to high-flow air and oxygen, using a pressure wire (Radi, St Jude Medical, Uppsala, Sweden). The mean transit time of a room temperature $3-4 \mathrm{ml}$ bolus of saline was recorded at rest and hyperaemic (adenosine) stress for both inspired gases. Resistance Index (RI), Resistance Reserve Index (RRI), Index of Microvascular Resistance (IMR) and Coronary Flow Reserve (CFR) were derived.

Results In volunteers the change in BOLD Air-T2* was $6.1 \pm$ $7.5 \mathrm{~ms}$ vs. $\mathrm{O}_{2}-\mathrm{T} 2 * 7.8 \pm 8.1, \mathrm{P}=0.575$. Rest Air-MBF was $0.88 \pm 0.40 \mathrm{ml} / \mathrm{min} / \mathrm{g}$ vs. $\mathrm{O}_{2}-\mathrm{MBF} 0.95 \pm 0.45, \mathrm{P}=0.168$ and hyperaemic Air-MBF $3.46 \pm 1.67$ vs. $\mathrm{O}_{2}-\mathrm{MBF} 3.35 \pm$ 2.05, $\mathrm{P}=0.821$.

In patients, ischaemic region change in BOLD Air-T2* was $1.1 \pm 6.6 \mathrm{~ms}$ vs. $\mathrm{O}_{2}-\mathrm{T} 2 * 2.7 \pm 8.2 \mathrm{~ms}, \mathrm{P}=0.583$ compared to Air-T2* $11.2 \pm 8.7$ vs. $\mathrm{O}_{2}-\mathrm{T} 2 * 2.9 \pm 2.5, \mathrm{P}=0.004$ in remote myocardium. Remote Air-MBF was $0.81 \pm 0.19$ vs. $\mathrm{O}_{2}-\mathrm{MBF} 0.76 \pm 0.15, \mathrm{P}=0.087$ and hyperaemic Air-MBF $1.31 \pm 0.047$ vs. $\mathrm{O}_{2}-\mathrm{MBF} 1.50 \pm 0.53, \mathrm{P}=0.249$; whilst ischaemic region Air-MBF was $0.83 \pm 0.20$ vs. $\mathrm{O}_{2}-\mathrm{MBF} 0.85$ $\pm 0.28, \mathrm{P}=0.725$ and hyperaemic Air-MBF $1.26 \pm 0.055$ vs. $\mathrm{O}_{2}-\mathrm{MBF} 1.35 \pm 0.59, \mathrm{P}=0.407$. Rest ischaemic Air-E was $0.69 \pm 0.14$ vs. $\mathrm{O}_{2}-\mathrm{E} 0.66 \pm 0.17, \mathrm{P}=0.408$ and remote Air-E was $0.73 \pm 0.13$ vs. $\mathrm{O}_{2}-\mathrm{E} 0.64 \pm 0.15, \mathrm{P}=$ 0.044 . Ischaemic region Air-PS was $0.67 \pm 0.38 \mathrm{ml} / \mathrm{min} / \mathrm{g}$ vs. $\mathrm{O}_{2}$-PS $0.58 \pm 0.32, \mathrm{P}=0.227$; whilst remote region Air-PS was $0.72 \pm 0.38$ vs. $\mathrm{O}_{2}$-PS $0.53 \pm 0.32, \mathrm{P}=0.024$.

Invasively there was an increase in rest transit time $0.69 \pm$ 0.35 vs. $0.97 \pm 0.50, \mathrm{P}=0.001$, resistance index $51.0 \pm$ 26.6 vs. $75.0 \pm 38.0, \mathrm{P}<0.001$, coronary flow reserve 2.21 \pm 1.30 vs. $2.90 \pm 1.51, \mathrm{P}=0.019$ and resistance reserve index $2.62 \pm 1.37$ vs. $3.54 \pm 1.74, \mathrm{P}=0.006$ in the air vs oxygen groups respectively.

Conclusion Supplemental high-flow oxygen (and hence hyperoxaemia) results in increased microvascular resistance in patients with severe $\mathrm{CAD}$, demonstrated during invasive coronary physiology studies. We have also shown with novel CMR techniques that, in patients with severe CAD, whilst there is no difference in absolute myocardial blood flow, high-flow supplemental oxygen results in a reduction in first-pass extraction fraction and capillary permeability-surface area product. This results in a reduction in myocardial oxygenation in the remote, non-ischaemic, myocardium. Supplemental high-flow oxygen should therefore be avoided in patients who are not hypoxic.

\section{E MICRORNA-140-5P AND SMURF1 REGULATE PULMONARY ARTERIAL HYPERTENSION}

\begin{abstract}
1,2Alexander MK Rothman*, 'Nadine D Arnold, 'Josephine A Pickworth, 'James Iremonger, ${ }^{3}$ Loredana Ciuclan, ${ }^{3}$ Robert Allen, ${ }^{4}$ Sabine Guth-Gundel, ${ }^{5}$ Mark Southwood, ${ }^{5}$ Nicholas W Morrell, ${ }^{6}$ Matthew Thomas, ${ }^{1}$ Sheila E Francis, ${ }^{3}$ David J Rowlands, ${ }^{1}$ Allan Lawrie. ${ }^{1}$ Department of Cardiovascular Science, University of Sheffield, Sheffield, UK; ${ }^{2}$ Sheffield Teaching Hospitals NHS Trust, Sheffield, UK; ${ }^{3}$ Novartis Institutes for Biomedical Research, Cambridge, MA, USA; ${ }^{4}$ Novartis Institutes for Biomedical Research, Basel, Switzerland; ${ }^{5}$ Department of Medicine, University of Cambridge School of Clinical Medicine, Addenbrooke's and Papworth Hospitals, Cambridge, UK; ${ }^{6}$ AstraZeneca R\&D, Respiratory, Inflammation and Autoimmunity (RIA) Innovative Medicines, Building AC461, SE-431 83 Mölndal, Sweden; *Presenting Author
\end{abstract}

\subsection{6/heartjil-2016-309890.224}

Background Clinical therapies for the treatment of pulmonary arterial hypertension (PAH) target vasoconstriction. However, the proliferative pulmonary vascular remodelling that drives disease persists contributing to significant patient morbidity and mortality. MicroRNA (miR) are short non-coding RNA that mediate post-transcriptional regulation of mRNA targets. We hypothesise that dysregulation of miR leads to de-repression of cellular targets central to disease pathogenesis.

Objective To identify dysregulated circulating $\mathrm{miR}$ in patients with $\mathrm{PAH}$, determine their phenotypic effect using in vitro and in vivo models and identify key mechanistic regulators that may represent novel therapeutic targets.

Methods and results Expression of miR-140-5p was reduced in whole blood samples from patients with PAH. Reduced expression of miR-140-5p at the time of diagnosis identified patients with adverse clinical characteristics. Transfection with miR-140-5p inhibitor resulted in increased proliferation PASMC and de-repression of key targets. Nebulised delivery of miR-140-5p mimic attenuated progression of established $\mathrm{PAH}$ in the SuHx rat model. Network and pathway analysis identified SMAD Specific E3 Ubiquitin Protein Ligase 1 (SMURF1) as a key miR-140-5p target. Whole blood mRNA and pulmonary vascular immunoreactivity of SMURF1 was increased in patients with PAH implicating SMURF1 in the pathology of human disease. Direct regulation of SMURF1 by miR-140-5p was demonstrated in-vitro by 3'UTR luciferase activity. Both miR-140-5p mimic and SMURF1 siRNA increased BMP response element activity identifying SMURF1 as a key negative regulator of BMP signalling in PASMC. Genetic ablation of SMURF1 in C57BL6 mice conferred allele dependent protection from SuHx induced PAH.

Conclusions These studies suggest that miR-140 and SMURF1 are key regulators of BMP signalling and disease pathology in $\mathrm{PAH}$ and highlight SMURF1 as a potential novel therapeutic target. 